\title{
The Nuclear Spectroscopic Telescope Array (NuSTAR)
}

Fiona A. Harrison ${ }^{a}$, Steve Boggs ${ }^{b}$, Finn Christensen $^{c}$, William Craig ${ }^{b, l}$, Charles Hailey ${ }^{e}$, Daniel Stern $^{f}$, William Zhang ${ }^{g}$, Lorella Angelini ${ }^{g}$, HongJun An $^{e}$, Varun Bhalereo ${ }^{a}$, Nicolai Brejnholt ${ }^{c}$, Lynn Cominsky ${ }^{h}$, W. Rick Cook ${ }^{a}$, Melania Dolle ${ }^{e}$ Paolo Giommi ${ }^{i}$, Brian Grefenstette ${ }^{a}$, Allan

Hornstrup $^{c}$, Vicky Kaspi ${ }^{j}$, Yunjin $\operatorname{Kim}^{f}$, Takeo Kitaguchi ${ }^{a}$, Jason Koglin ${ }^{e}$,

Carl Christian Liebe $f$, Greg Madejski ${ }^{k}$, Kristin Kruse Madsen ${ }^{a}$, Peter Mao ${ }^{a}$ David Meier ${ }^{f}$, Hiromasa Miyasaka ${ }^{a}$, Kaya Mori ${ }^{e}$, Matteo Perri ${ }^{i}$, Michael Pivovaroff ${ }^{l}$, Simonetta Puccetti ${ }^{i}$, Vikram Rana ${ }^{a}$, Andreas Zoglauer ${ }^{b}$

${ }^{a}$ Caltech Division of Physics, Mathematics and Astronomy, Pasadena, USA;

${ }^{b}$ U.C. Berkeley Space Sciences Laboratory, Berkeley, CA;

${ }^{c}$ Danish Technical University Space Center, Copenhagen, DK ;

${ }^{e}$ Columbia University, NY, USA;

${ }^{f}$ Jet Propulsion Laboratory, Pasadena, CA, USA;

${ }^{g}$ Goddard Space Flight Center, Greenbelt, MD, USA;

${ }^{h}$ Sonoma State University, Sonoma, CA, USA;

${ }^{i}$ ASI Science Data Center, Rome, IT ;

${ }^{j}$ McGill University, Montreal, CA;

${ }^{k}$ Stanford University, SLAC, USA;

${ }^{l}$ Lawrence Livermore National Laboratory, Livermore, CA, USA;

\begin{abstract}
The Nuclear Spectroscopic Telescope Array (NuSTAR) is a NASA Small Explorer mission that will carry the first focusing hard X-ray $(6-80 \mathrm{keV})$ telescope to orbit. NuSTAR will offer a factor $50-100$ sensitivity improvement compared to previous collimated or coded mask imagers that have operated in this energy band. In addition, $N u S T A R$ provides sub-arcminute imaging with good spectral resolution over a 12-arcminute field of view. After launch, $N u S T A R$ will carry out a two-year primary science mission that focuses on four key programs: studying the evolution of massive black holes through surveys carried out in fields with excellent multiwavelength coverage, understanding the population of compact objects and the nature of the massive black hole in the center of the Milky Way, constraining the explosion dynamics and nucleosynthesis in supernovae, and probing the nature of particle acceleration in relativistic jets in active galactic nuclei. A number of additional observations will be included in the primary mission, and a guest observer program will be proposed for an extended mission to expand the range of scientific targets. The payload consists of two co-aligned depth-graded multilayer coated grazing incidence optics focused onto a solid state CdZnTe pixel detectors. To be launched in early 2012 on a Pegasus rocket into a low-inclination Earth orbit, NuSTAR largely avoids SAA passage, and will therefore have low and stable detector backgrounds. The telescope achieves a 10.14-meter focal length through on-orbit deployment of an extendable mast. An aspect and alignment metrology system enable reconstruction of the absolute aspect and variations in the telescope alignment resulting from mast flexure during ground data processing. Data will be publicly available at GSFC's High Energy Archive Research Center (HEASARC) following validation at the science operations center located at Caltech.
\end{abstract}

Keywords: X-rays, gamma-rays, missions

Further author information: (Send correspondence to F.A.H.) F.A.H.: E-mail: fiona@srl.caltech.edu

Space Telescopes and Instrumentation 2010: Ultraviolet to Gamma Ray,

edited by Monique Arnaud, Stephen S. Murray, Tadayuki Takahashi, Proc. of SPIE Vol. 7732,

77320S · C 2010 SPIE · CCC code: 0277-786X/10/\$18 - doi: 10.1117/12.858065

Proc. of SPIE Vol. 7732 77320S-1 


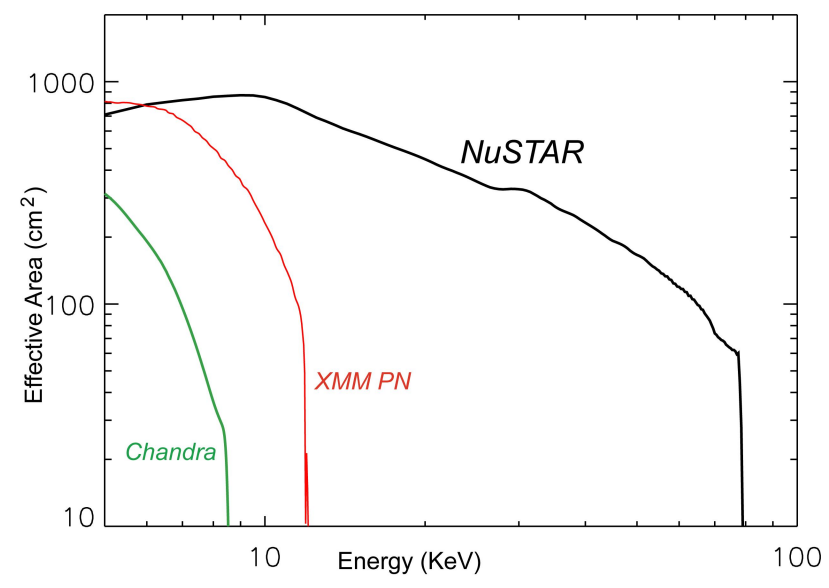

Figure 1. Effective area for two telescopes as a function of energy compared with Chandra and XMM. NuSTAR utilizes a low graze angle design combined with depth-graded multilayer coatings to extend sensitivity to $80 \mathrm{keV}$.

\section{INTRODUCTION}

The last decade has seen a major technological advance in hard X-ray/soft gamma-ray astronomy - the ability to focus efficiently - enabling instruments that improve sensitivity by orders of magnitude compared to the collimators and coded-mask cameras previously used to observe the cosmos at these energies. Focusing instruments achieve large concentration factors, such that their collecting area is significantly larger (by factors of 1000 or greater) than the detector area used to register the signal. In the hard X-ray band, where particle interactions result in high detector backgrounds, large concentration factors result in enormous improvements in the signal to background ratio over coded mask cameras, where telescope effective areas are typically less than $(\sim 50 \%)$ of the detector area. Focusing telescopes operating at energies above $10 \mathrm{keV}$ have recently been developed and deployed on balloon platforms, ${ }^{1-3}$ and will be incorporated on two approved space experiments; The Nuclear Spectroscopic Telescope Array NASA Small Explorer, the JAXA Astro- $H^{4}$ mission, as well as the proposed New Hard X-ray Mission (NHXM). ${ }^{5}$

Extending focusing to the hard X-ray band requires a combination of low graze-angle and/or depth-graded multilayer coated optics combined with imaging detectors utilizing high-atomic-number materials. For grazingincidence X-ray optics, the graze angle (angle at which X-rays are reflected from a shell) range for which efficient reflectance can be achieved scales approximately inversely with energy. To achieve high-energy $(10-60 \mathrm{keV})$ response with traditional metal coatings the optics design must utilize graze angles of a few arcminutes or less, which requires small-radius, tightly-nested optics shells. Since the field of view of a grazing incidence telescope is approximately equal to the average graze angle, metal-coated optics will have small fields of view at high energy. To overcome this limitation, a number of future astronomical telescopes will employ depthgraded multilayer coatings, ${ }^{6}$ which exploit the principal of Bragg reflection to increase the graze angles at which significant reflectance can be achieved. This enables high throughput with moderate $\left(\sim 10^{\prime}\right)$ FoV.

The NuSTAR Small Explorer mission will be the first astronomical telescope on-orbit to utilize the new generation of hard X-ray optics and detector technologies to carry out high-sensitivity observations at X-ray energies significantly greater than $10 \mathrm{keV}$. NuSTAR, based in large part on the technologies developed for the High-Energy Focusing Telescope $(H E F T)^{1}$ balloon experiment, was selected after a competitive Phase A study for implementation, and the mission is now in Phase D with a launch scheduled for the first part of calendar year 2012. This paper describes NuSTAR's two-year primary science program, the implementation of the science instrument, the mission design, and plans for data distribution and archiving.

\section{SCIENTIFIC PERFORMANCE}

NuSTAR will fly two co-aligned focusing hard X-ray telescopes consisting of multilayer coated grazing-incidence optics and shielded solid state CdZnTe pixel detectors with a 10.14-meter focal length. Figure 1 shows the total 
Table 1. Key instrument performance parameters.

\begin{tabular}{|l|c|}
\hline Energy range & $5-80 \mathrm{keV}$ \\
\hline Angular resolution (HPD) & $45^{\prime \prime}$ \\
\hline FoV $(50 \%$ resp.) at $10 \mathrm{keV}$ & $10^{\prime}$ \\
\hline FoV $(50 \%$ resp.) at $68 \mathrm{keV}$ & $6^{\prime}$ \\
\hline Sensitivity $(6-10 \mathrm{keV})\left[10^{6} \mathrm{~s}, 3 \sigma, \Delta \mathrm{E} / \mathrm{E}=0.5\right]$ & $2 \times 10^{-15} \mathrm{erg} / \mathrm{cm}^{2} / \mathrm{s}$ \\
\hline Sensitivity $(10-30 \mathrm{keV})\left[10^{6} \mathrm{~s}, 3 \sigma, \Delta \mathrm{E} / \mathrm{E}=0.5\right]$ & $1 \times 10^{-14} \mathrm{erg} / \mathrm{cm}^{2} / \mathrm{s}$ \\
\hline Background in HPD $(10-30 \mathrm{keV})$ & $6.8 \times 10^{-4} \mathrm{cts} / \mathrm{s}$ \\
\hline Background in HPD $(30-60 \mathrm{keV})$ & $4.0 \times 10^{-4} \mathrm{cts} / \mathrm{s}$ \\
\hline Spectral resolution $(\mathrm{FWHM})$ & $500 \mathrm{eV}$ at $10 \mathrm{keV}, 1.2 \mathrm{keV}$ at $80 \mathrm{keV}$ \\
\hline Strong source $(>10 \sigma)$ positioning & $1.5^{\prime \prime}(1-\sigma)$ \\
\hline Temporal resolution & $2 \mu \mathrm{sec}$ \\
\hline Target of Opportunity response & $<24 \mathrm{hours}$ \\
\hline
\end{tabular}

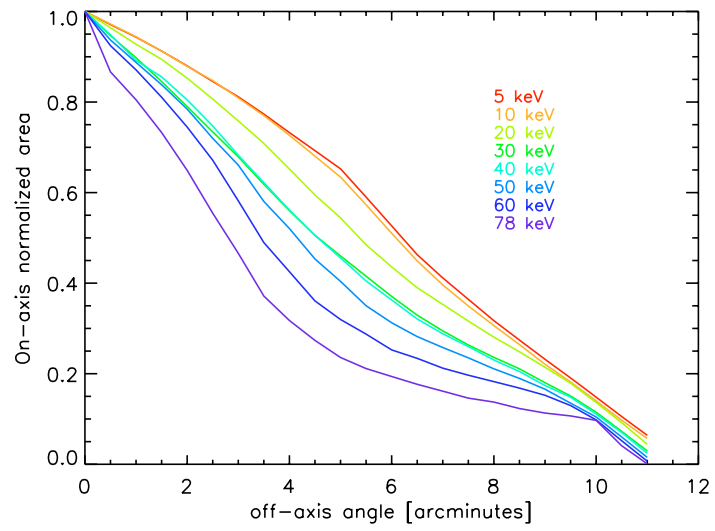

Figure 2. Effective area as a function of off-axis angle, as a fraction of on-axis area, for several energies.

effective area for both telescopes as a function of energy, with a comparison to Chandra and $X M M$. The energy band extends from about $5 \mathrm{keV}$ to $80 \mathrm{keV}$, being limited at the low-energy end by the optics thermal cover and shield entrance window, and at the high energy end by the K-edge (at $78.4 \mathrm{keV}$ ) in the Platinum mirror coatings.

Table 1 provides the key instrument performance parameters. The $45^{\prime \prime}$ angular resolution is projected based on mechanical metrology of the first flight optic, which is currently more than $75 \%$ complete, combined with a model of contributions from on-orbit thermal distortions and aspect reconstruction. The field if view is energydependent due to changes in multilayer reflectance as a function of energy and optics shell radius, which results in overall loss of reflectance and more vignetting at high energy (see Figure 2). The spectral resolution is $500 \mathrm{eV}$ at energies below $\sim 30 \mathrm{keV}$, and increases to $1.2 \mathrm{keV}$ at the upper end of the energy range. The $2 \mu$ sec temporal resolution, determined by the bit rate allocated in the telemetry stream for time tags, is more than adequate to meet scientific requirements. The intrinsic temporal resolution of the detector is better than $1 \mu \mathrm{sec}$. The target of opportunity (ToO) response time is required to be less than 24 hours, however on average the turnaround will be faster, with targets acquired within 6 hours.

\section{BASELINE MISSION SCIENCE PLAN}

The two-year NuSTAR baseline science mission begins after a 1-month on orbit checkout period. During this time $N u S T A R$ will focus on a set of four key objectives (Table 2). If the currently-estimated performance is achieved 
Table 2. Core science program.

\begin{tabular}{|l|l|}
\hline Key science goal & Observations \\
\hline \hline Locate massive black holes & $\begin{array}{l}\text { Deep and wide-field surveys } \\
\text { in GOODS, COSMOS, XBootes }\end{array}$ \\
\hline Study the population of compact objects in the Galaxy & Galactic survey centered on Sgr A* \\
\hline $\begin{array}{l}\text { Understand explosion dynamics and nucleosynthesis in } \\
\text { core collapse and Type1a SNe }\end{array}$ & $\begin{array}{l}\text { Pointed observations of Cas A, SN1987A, Tycho } \\
\text { ToO observations of 1a SNe }\end{array}$ \\
\hline $\begin{array}{l}\text { Constrain particle acceleration in relativistic jets } \\
\text { in supermassive black holes }\end{array}$ & $\begin{array}{l}\text { Contemporaneous multiwavelength observations } \\
\text { of GeV and TeV blazars }\end{array}$ \\
\hline
\end{tabular}

on-orbit, completing these objectives will require about eighteen months of observation time. The remaining six months will be used to perform additional targeted programs. Although survey fields and specific sources for the key programs have been identified, the detailed designs of the observations are not yet complete. The science team is also in the process of prioritizing the additional science objectives. The final baseline science observing plan will be determined six months prior to launch, although alterations may be made after launch as a result of measured on-orbit performance or preliminary data analysis.

Table 2 summarizes the key objectives and the associated observations. The primary extragalactic science objective is to understand the physical processes that drive the evolution of supermassive black holes and galaxies at redshift $z \lesssim 1$. NuSTAR's specific contribution will be to measure the evolution of obscured active galactic nuclei (AGN), study the characteristics of the galaxies that host them, and determine if they evolve similarly to the well-studied unobscured population. In addition, NuSTAR will undertake simultaneous observations of blazar AGN with Fermi and the TeV gamma-ray telescopes Veritas and HESS, as well as with ground-based optical and radio telescopes in order to study particle acceleration in jets. In the Galaxy, NuSTAR will advance the understanding of explosion dynamics and nucleosynthesis in supernovae by mapping Cas $\mathrm{A}$ in the radioactive decay of ${ }^{44} \mathrm{Ti}(68 \mathrm{keV})$. Other remnants that will be studied include SN 1987A, Tycho, and G1.9+03. NuSTAR will also survey regions of the Galaxy, with the primary survey field being the few square degrees centered on $\operatorname{Sgr} A^{*}$, a region that contains $1 \%$ of the stellar mass, but $10 \%$ of the massive young stars. The Galactic surveys will identify hundreds of compact stellar remnants even in obscured regions, and will map diffuse features associated with molecular cloud complexes. The NuSTAR mission is designed to have access to $80 \%$ of the sky at any time, and has target of opportunity capability to enable followup of any Type 1a supernova out to Virgo or core collapse in the local group that occurs during the mission life.

A wide variety of programs are being considered to fill the science reserve time. These include observations of the sun to search for microflares believed to heat the corona, high signal to noise spectroscopy of bright AGN, surveys and ToO observations of known magnetars, mapping non-thermal emission in galaxy clusters, and imaging of nearby starburst galaxies. While a number of these programs will be accomplished in the two-year baseline mission, others will be good candidates for Guest Observer (GO) proposals undertaken in an extended mission. NuSTAR has no consumables, and the expected orbit lifetime is in excess of five years. The team expects to propose a GO program to the NASA Senior Review that will broaden the scientific return and enable broader community participation.

\section{SCIENCE INSTRUMENT IMPLEMENTATION}

The NuSTAR science instrument (see Figure 3) consists of two co-aligned grazing incidence optics focusing on to two shielded solid state CdZnTe pixel detectors. The instrument is launched in a compact, stowed configuration, and after launch a 10-meter mast, manufactured by ATK Space Systems, Goleta, is deployed to achieve a focal length of $10.14 \mathrm{~m}$. Because the absolute deployment location of the mast is difficult to measure on the ground, due to complications associated with complete gravity offloading, an adjustment mechanism is built into the last section of the mast to enable a one-time alignment to optimize the location of the optical axes on the focal plane. This mechanism provides two angular adjustments as well as rotation. The mast is not perfectly rigid, but undergoes thermal distortions particularly when going in and out of Earth shadow (the mission is deployed 


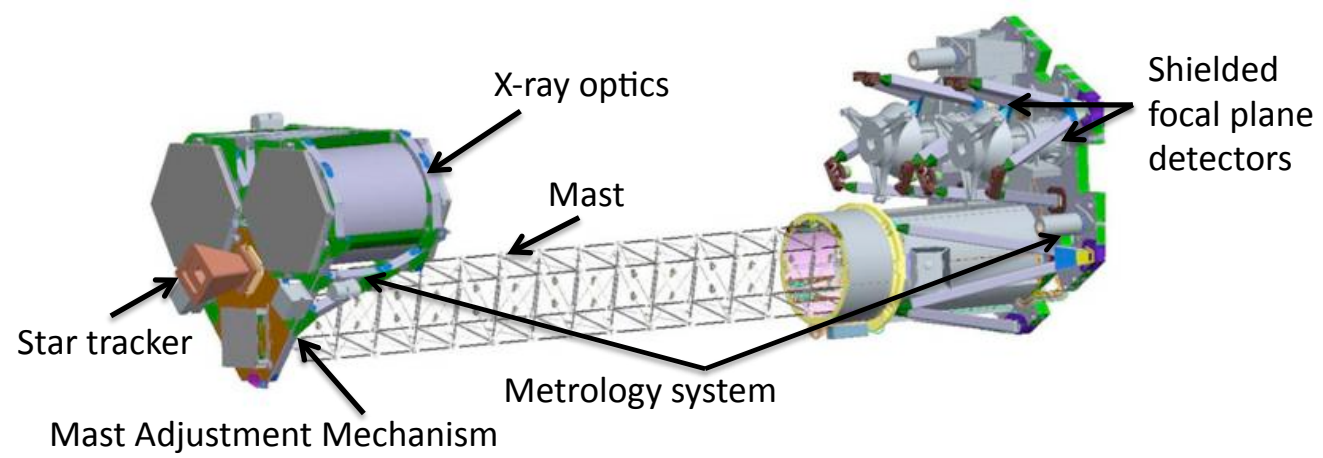

Figure 3. Diagram of the NuSTAR instrument showing the principal elements.

in a low-Earth orbit) that translate into changes in telescope alignment of $1-2^{\prime}$. These mast alignment changes are measured by the combination of an optics bench-mounted star tracker and a laser metrology system. The same combination of sensors also provides the absolute instrument aspect. In order to limit the field of view open to the detectors, and therefore the diffuse cosmic background, an aperture stop consisting of three rings deploys with the mast. The aperture stop is not shown deployed in Figure 3.

Table 3. Optics configuration summary.

\begin{tabular}{|l|c||l|c|}
\hline Parameter & Value & Parameter & Value \\
\hline \hline focal length & $10.14 \mathrm{~m}$ & shell length & $22.5 \mathrm{~cm}$ \\
\hline \# shells & 130 & min. graze angle & $1.34 \mathrm{mrad}$ \\
\hline \# azimuthal segments & 6 (inner) $/ 12($ outer) & max. graze angle & $4.7 \mathrm{mrad}$ \\
\hline inner radius & $5.44 \mathrm{~cm}$ & coating (outer) & $\mathrm{W} / \mathrm{Si}$ \\
\hline outer radius & $19.1 \mathrm{~cm}$ & coating (inner) & $\mathrm{Pt} / \mathrm{C}$ \\
\hline
\end{tabular}

The NuSTAR optics utilize a conical approximation to a Wolter-I design in a highly-nested configuration, with 130 shells per optic with graze angles ranging from 4.6 to 16 arcminutes. The shells are fabricated from segmented thermally formed glass, and the segments are coated with depth-graded multilayers optimized to achieve significant high energy response for this graze angle range. ${ }^{7}$ The coatings employ a combination of $\mathrm{W} / \mathrm{Si}$ bilayers on the outer shells, and $\mathrm{Pt} / \mathrm{C}$ on the inner shells. The high-energy effective area cutoff results from the K-shell absorption in Platinum. Hailey et al. (2010) describe the optics implementation in detail, and Table 3 provides a summary of the primary configuration parameters.

The focal plane consists of four CdZnTe pixel sensors coupled to a custom low-noise ASIC. ${ }^{9}$ Each hybrid contains a $32 \times 32$ array of $600 \mu \mathrm{m}$ pixels with a resulting plate scale of $12.3^{\prime \prime} /$ pixel, so that the mirror point spread function is over sampled. The sensors are placed in a two-by-two array with a minimal $(\sim 500 \mu \mathrm{m})$ gap between them to fill a total subtended field of view of $13^{\prime}$ on a side (Figure 4). Table 4 summarizes the primary characteristics of the focal plane.

Table 4. Focal plane configuration summary.

\begin{tabular}{|l|c||l|c|}
\hline Parameter & Value & Parameter & Value \\
\hline \hline pixel size & $0.6 \mathrm{~mm} / 12.3^{\prime \prime}$ & max processing rate & $400 \mathrm{evt} / \mathrm{s}$ \\
\hline focal plane size & $13^{\prime} \times 13^{\prime}$ & max flux meas. rate & $10^{4} / \mathrm{s}$ \\
\hline pixel format & $32 \times 32$ & time resolution & $2 \mu \mathrm{sec}$ \\
\hline threshold & $2.5 \mathrm{keV}($ each pixel) & dead time fraction (weak source) & $2 \%$ \\
\hline
\end{tabular}



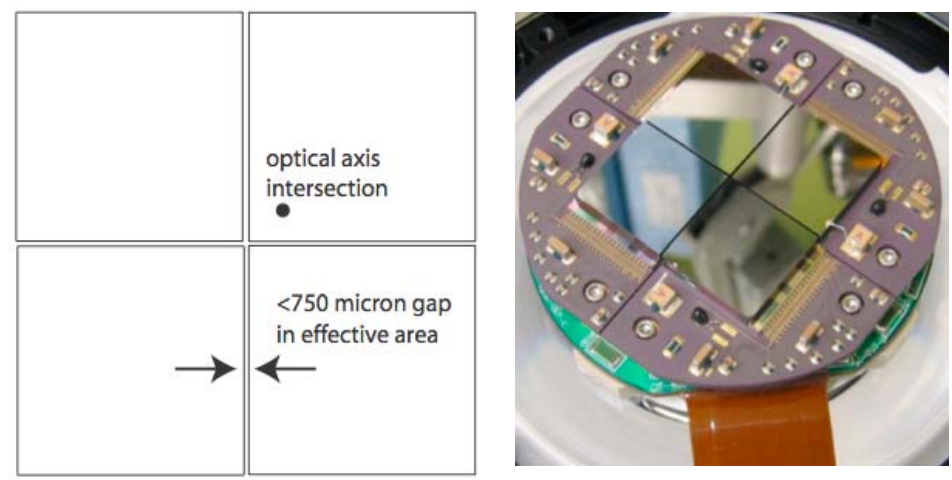

Figure 4. The NuSTAR focal plane configuration and photograph of an engineering test module.

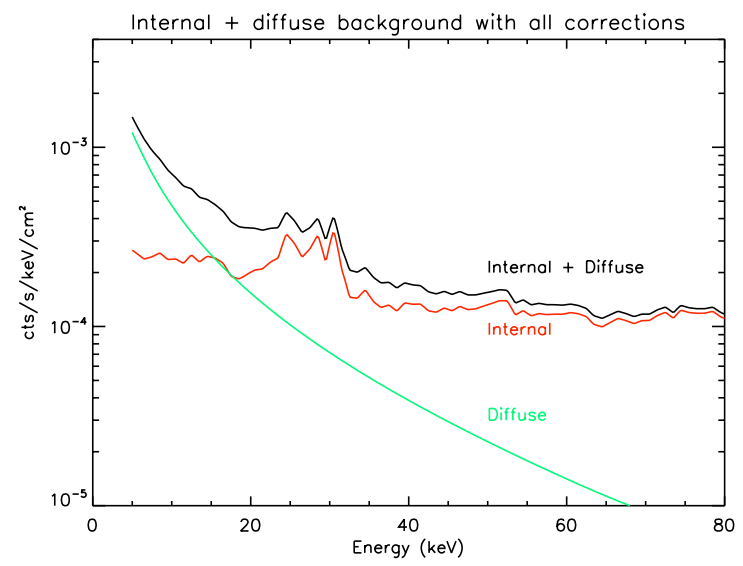

Figure 5. Predicted detector background count rate per unit area as a function of energy.

To achieve a low energy threshold and good spectral performance the detector readout is designed for very low noise. The electronic noise contribution (including detector leakage current) to the energy resolution is $400 \mathrm{eV}$, and the low-energy threshold is $2.5 \mathrm{keV}$ for an event registering in a single pixel. Over most of the energy range the detector spectral resolution is limited by charge collection uniformity in the CdZnTe crystal. At low energies, between 5 and $30 \mathrm{keV}$, the average spectral resolution for a typical flight detector is $500 \mathrm{eV}$ FWHM, while at $60 \mathrm{keV}$ it is $1.0 \mathrm{keV}$, and at $86 \mathrm{keV} 1.2 \mathrm{keV}$ FHWM. The focal plane will be passively cooled in flight to between $0^{\circ}$ and $5^{\circ} \mathrm{C}$. The passive cooling is enabled by the low-power dissipation of the detector readout chip $(50 \mu \mathrm{W} /$ pixel $)$. At the in-flight operating temperatures detector leakage current is a negligible contributor to the resolution. In addition to measuring the deposited energy and arrival time for each event, the readout architecture enables a depth of interaction measurement which can be used both to maximize photo peak efficiency at high-energy, where charge trapping effects can lead to a low-energy 'tail' on the energy resolution, and in addition reject background from the back portion of the detector.

The readout of each focal plane module is controlled by an FPGA-embedded microprocessor. The maximum rate that events can be processed is $400 \mathrm{cps}$ in each telescope, however pulse pileup does not occur until substantially higher rates $\left(\sim 10^{5} \mathrm{cps}\right)$. The readout system is designed so that source fluxes can be measured up to countrates of $10^{4} \mathrm{cps}$. At the nominal faint-source count rates the readout dead time is $\lesssim 2 \%$. 
The focal plane is surrounded by an active $2 \mathrm{~cm}$ thick $\mathrm{CsI}(\mathrm{Na})$ shield and incorporates an deployable aperture stop. The CsI shield extends $20 \mathrm{~cm}$ above the detector, and has an opening angle of 16 degrees, while the passive aperture stop defines a much narrower opening of 4 degrees. Figure 5 shows the expected background counts per unit detector area as calculated using the GEANT-based MGGPOD ${ }^{10}$ Monte Carlo suite. At low energies the background is dominated by diffuse leakage through the portion of the aperture stop field of view not blocked by the optics bench. The spectral features between 25 and $35 \mathrm{keV}$ are fluorescence from the CsI shield. The background level shown in Figure 5 assumes the use of the depth-of-interaction measurement to reject interactions in the back of the detector, which results in about a factor two background reduction at $60 \mathrm{keV}$.

The instrument components are in a mature state, with flight hardware fabrication underway, and delivery to integration and test beginning in Fall 2010.

\section{MISSION DESIGN}

NuSTAR will be launched on a Pegasus XL rocket into a $6^{\circ}$ inclination, $575 \times 600 \mathrm{~km}$ low Earth orbit. The low inclination, achieved by using a near-Equatorial launch site at Kwajalein Island, significantly reduces internal detector backgrounds because the orbit avoids the intense region of the SAA (which it still skims on some orbits). The orbit altitude leads to an expected mission life in excess of five years. The telescope will not re-orient during Earth occultations, and the total expected observing efficiency is $50 \%$ for typical targets, with higher efficiency (approaching 90\%) for targets near the poles. The spacecraft, manufactured by Orbital Sciences Corp., is threeaxis stabilized with a single articulating solar panel and predominantly relies about a multi-head star camera (the DTU microASC) for aspect. This enables $80 \%$ of the sky to be accessed at any given time, which both allows $\mathrm{ToO}$ viewing with few restrictions, and aids in mission planning.

The satellite will be operated out of the Mission Operations Center at U.C. Berkeley. Command uplinks and data downlinks will be through a ground station, operated by the Agenzia Spaziale Italiana, located in Malindi, Kenya. Most science targets will be viewed for a week or more, so that after a 30-day in-orbit checkout and commissioning period, commanding will be rare. The turnaround time for target of opportunity observations depends largely on timing relative to the ground station passes. The Malindi station is visible once per 90 minute orbit, but commands can take up to 12-hours to prepare given that the MOC is not staffed 24 hours/day. The spacecraft slews at an average rate of $1.2^{\circ} /$ minute, so a typical slew will take less than 90 minutes.

\section{GROUND DATA SYSTEM}

The science data will be transferred from the U.C. Berkeley MOC to a Science Operations Center located at Caltech. The SOC will process and validate the data, and distribute products to the science team. All science data will be converted to FITS format conforming to OGIP standards, and analysis software will adopt the FTOOLS approach and environment. The NUSTAR science data has no proprietary period, and after a sixmonth interval during which the instrument calibration will be understood and the performance verified, data will enter the public science archive, located at the High Energy Archive Science Center at Goddard Space Flight Center, within two months of completion of an observation (the two-month period being required for ground data processing and validation).

\section{SUMMARY AND CONCLUSIONS}

With a launch scheduled for early 2012, NuSTAR will be the first of a new generation of focusing hard Xray telescopes, and will provide two orders of magnitude improvement in sensitivity over previous hard X-ray missions, combined with good spectral resolution and sub-arcminute imaging. In the first two years $N u S T A R$ will focus on four key science objectives, however the mission life is limited only by the orbit decay, and an extended mission will be proposed to allow a substantial guest investigator program to expand the scientific reach. The scientific performance expectations are largely based on measurements made on prototype and flight hardware, giving confidence in current projections for sensitivity, spectroscopic and imaging performance.

$N U S T A R$ will incorporate a number of recently-developed technologies. At the heart of the instrument are novel depth-graded multilayer optics focusing on to CdZnTe pixel detectors developed originally for the HEFT 
balloon experiment. NuSTAR will be the first X-ray telescope to utilize a long (10-meter) extendable mast, and will demonstrate the application of this technology as well as the requisite metrology system. While the instrument contains new elements, the spacecraft is based on a heritage design, and the mission operations approach is simple. These elements enable a highly-capable science mission on a Small Explorer platform.

\section{ACKNOWLEDGMENTS}

The NuSTAR mission is funded by NASA through contract number NNG08FD60C. Additional contributions are provided by the Danish Technical University for optics coating and calibration and the Agenzia Spaziale Italiana (ASI) for the Malindi ground station and ground data system development. Mission management is provided by the Jet Propulsion Laboratory, and program management provided by the Explorer Program Office at Goddard Space Flight Center.

\section{REFERENCES}

[1] Harrison, F. A., Christensen, F. E., Craig, W., Hailey, C., Baumgartner, W., Chen, C. M. H., Chonko, J., Cook, W. R., Koglin, J., Madsen, K.-K., Pivavoroff, M., Boggs, S., and Smith, D., "Development of the HEFT and NuSTAR focusing telescopes," Experimental Astronomy 20, 131-137 (Dec. 2005).

[2] Ramsey, B. D., Alexander, C. D., Apple, J. A., Benson, C. M., Dietz, K. L., Elsner, R. F., Engelhaupt, D. E., Ghosh, K. K., Kolodziejczak, J. J., O'Dell, S. L., Speegle, C. O., Swartz, D. A., and Weisskopf, M. C., "First Images from HERO, a Hard X-Ray Focusing Telescope," ApJ 568, 432-435 (Mar. 2002).

[3] Tueller, J., Krimm, H. A., Okajima, T., Barthelmy, S. D., Owens, S. M., Serlemitsos, P. J., Soong, Y., Chan, K.-W., Ogasaka, Y., Shibata, R., Tamura, K., Furuzawa, A., Tawara, Y., Kunieda, H., and Yamashita, K., "InFOC $\mu \mathrm{S}$ Hard X-ray Imaging Telescope," Experimental Astronomy 20, 121-129 (Dec. 2005).

[4] Takahashi, T. et al., "The ASTRO-H Mission," in [These proceedings], (2010).

[5] Pareschi, G., Tagliaferri, G., Attinà, P., Basso, S., Borghi, G., Citterio, O., Civitani, M., Cotroneo, V., Negri, B., Sironi, G., Spiga, D., Vernani, D., and Valsecchi, G., "Design and development of the optics system for the NHXM Hard X-ray and Polarimetric Mission," in [Society of Photo-Optical Instrumentation Engineers (SPIE) Conference Series], Society of Photo-Optical Instrumentation Engineers (SPIE) Conference Series 7437 (Aug. 2009).

[6] Christensen, F. E., Hornstrup, A., Westergaard, N. J., Schnopper, H. W., Wood, J., and Parker, K., "A graded d-spacing multilayer telescope for high energy X-ray astronomy," in [Society of Photo-Optical Instrumentation Engineers (SPIE) Conference Series], R. B. Hoover, ed., Society of Photo-Optical Instrumentation Engineers (SPIE) Conference Series 1546, 160-167 (Jan. 1992).

[7] Madsen, K. K., Harrison, F. A., Mao, P. H., Christensen, F. E., Jensen, C. P., Brejnholt, N., Koglin, J., and Pivovaroff, M. J., "Optimizations of $\mathrm{Pt} / \mathrm{SiC}$ and W/Si multilayers for the Nuclear Spectroscopic Telescope Array," in [Society of Photo-Optical Instrumentation Engineers (SPIE) Conference Series], Society of PhotoOptical Instrumentation Engineers (SPIE) Conference Series 7437 (Aug. 2009).

[8] Hailey, C. J. et al., "The nuclear spectroscopic telescope array (NuSTAR): optics overview and current status," in [These proceedings], (2010).

[9] Harrison, F. A. and Cook, W. R. and Miyasaka, H. and McLean, R., [Semiconductor radiation detection systems], 67, CRC Press (2010).

[10] Weidenspointner, G., Sturner, S. J., Novikova, E. I., Harris, M. J., Zoglauer, A., Wunderer, C. B., Kippen, R. M., Bloser, P., and Zeitnitz, C., "MGGPOD: A Monte Carlo Suite for Gamma-Ray Astronomy Version 1.1," in [ESA Special Publication], ESA Special Publication 622, 637-+ (2007). 